\title{
E-LIS: Unique Model for Subject Specific Open Access Repository
}

\author{
Antonella De Robbio and Imma Subirats Coll
}

\begin{abstract}
E-LIS established in 2003 is an international open access archive on librarianship, information science and technology, and related disciplines. It uses the Open Archives Initiative (OAI) protocol and tools to facilitate interoperability between repository servers. E-LIS is the first e-server and largest repository in Library and Information Science (LIS) and is managed and maintained by an international team of experts working on a voluntary basis.

The purpose of this paper is to describe the main characteristics (technical and organizational) of the archive and its configuration and customisation, and discuss its policies, aims and mission. Its main focus, however, is on the E-LIS organizational model and on the strategic issues correlated to Open Access (OA). The challenges and opportunities of E-LIS consequent to a global vision for the LIS are also discussed. It explains how E-LIS stimulates participation from all over the world, which helps research activities on the subject worldwide. The paper emphasises that E-LIS can promote OA movement in general, and so it can help in disseminating the OA philosophy serving as a model to reveal the benefits of international subject specific open access repositories.
\end{abstract}

\section{Introduction}

One of the most recent developments in the field of Library and Information Science (LIS) is the trend towards digital libraries and self-archiving. Self-archiving can be defined as the deposit of a digital document in a public, free-access repository, for example, an e-print archive. An e-print archive is a collection of digital research documents such as articles, book chapters, conference papers and data sets. E-prints are the digital texts of peer-reviewed research articles, before and after refereeing. Before refereeing and publication, the draft is called a 'preprint'. The refereed, accepted, final draft is called a 'post print'. The term e-prints include both preprints and post prints.

E-LIS established in 2003 is an international, open access archive related to librarianship, information science and technology, computing and related disciplines. Its objectives are the same as those of the eprints movement (Lewandowski, 2004) and the Free Online Scholarship (FOS) movement (Suber 2012). E-LIS is now the biggest repository in LIS. Within a decade after its establishment, the collection has reached about 15000 documents, which consists of books, theses, research papers and presentations. The E-LIS model is based on community standards, which provide the mechanism for enforcement of proper attribution, and responsible use of published works in line with the two movements mentioned above. On the technical level, it is based on the Open Archives Initiative (OAI) and shares its standards and protocols. E-LIS is the first international e-server in this subject area and 
is part of the RCLIS (Research in Computing, Library and Information Science) project (RCLIS, 2012).

The purpose of the E-LIS archive is to make full-text documents visible, accessible, harvestable, searchable and usable by any potential user with access to the Internet. It also aims to support individuals who wish to publish or make their papers available worldwide. LIS communities in any country can use it. Works can be deposited in any language and format; authors can self-archive them; a proxy service supports the depositors. The archive is open to proposals from new partners and it has agreements with institutions and library and information science associations of various countries. ELIS is organised, managed and maintained by an international team of library and information science professionals working on a voluntary basis. The flexible architecture of the OAI is sub-divided into data provider and service provider. A data provider maintains one or more repositories (web servers) that support the OAI protocol as a means of exposing metadata. A service provider issues requests to data providers and uses the metadata as a basis for building value-added services. By self-archiving, authors can store a copy of their documents in a disciplinary or an institutional archive. As the OAI architecture enables documents to become swiftly available worldwide, selfarchiving helps to maximize the visibility and accessibility of refereed research, and hence to maximizing its usage by researchers and to enhancing its impact on research.

Self-archiving systems can be either centralised or distributed and are usually subject or institution-based. At present there are several subject-based archives, including ArXiv, CogPrints and E-LIS, which are centralised, and RePEC, which is distributed. There are many institution-based archives. But the advantage of subject-based archives, like E-LIS, is that they are specific to discipline, needs and requirements and to some extent quality control is also possible. Many

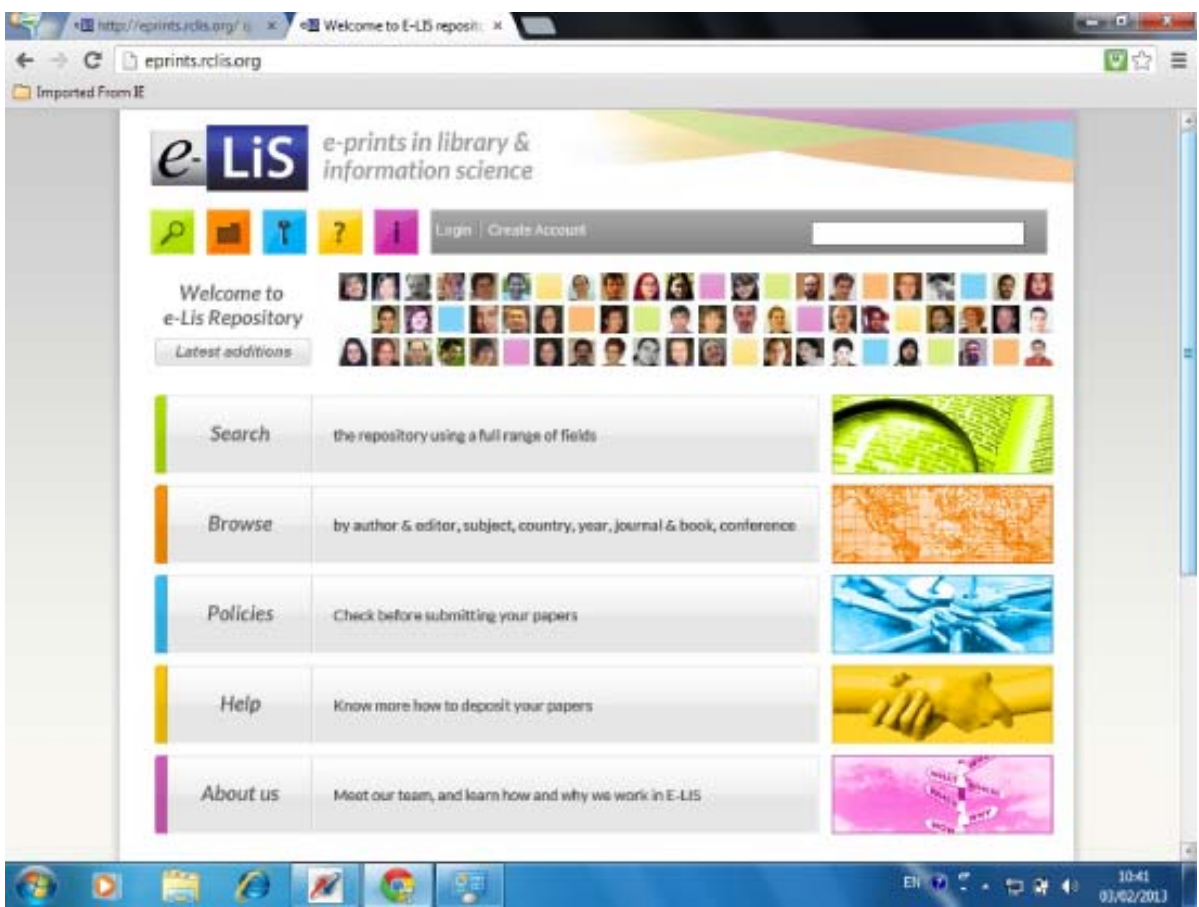

Figure 1: E-LIS Home Page 
repositories use the OAI protocol and tools to facilitate interoperability between repository servers.

The main characteristics of a centralised archive such as E-LIS are:

- Storage and distribution of data from a single location;

- Centralised access control over the supply and re-use of data;

- Checking, cleaning and processing of data according to standard criteria;

- Centralised support service for describing the contents of the data, defining the principles and practices governing the collection of data, and other relevant properties of the data;

- Cataloguing of technical and substantive properties of data for information retrieval and,

- User support after supply of data.

E-print repositories are complementary to, rather than a replacement for scholarly journals. The peer-review process provided by journals is of critical importance to scholarship. However, the proliferation of institution- and discipline-based e-print repositories could accelerate changes that are currently taking place in the scholarly communication process, which in turn could increase the number of journals moving from toll access (reader pays/subscriptionbased model) to open access (author-pays/ free access model).

The evolving academic discourse surrounding the concept of Open Access $(\mathrm{OA})$ provided the underlying motivation for the establishment of E-LIS. The library and information world is highly integrated with the areas of computing science and technology and it was felt that the LIS discipline should set an example to other communities by providing a state-of-the-art model for the OA movement and digital libraries, particularly in relation to the open archive model, within which E-LIS is a disciplinary repository. The extension of the OA concept to LIS works and the dissemination of material within the LIS community will contribute to the development of an international LIS network. Contributions of E-LIS to these two functions are unparalleled. For library and information science professionals Meta data creation is costly and the growing trend of authors to self-archive in the OAI framework is being proved to be an effective way to reduce some of those costs. For librarians as authors, archiving their work in E-LIS gives them an increased understanding of the process of selfarchiving and the E-LIS archive ensures data preservation and a wide data visibility. In addition it facilitates active participation in the international community of library and information science professionals.

E-LIS defines Open Access as a property of individual works and it adheres to the Bethesda Declaration (OAPC, 2003), which states that publication must meet two conditions:

1. The author(s) and copyright holder(s) grant(s) to all users a free, irrevocable, worldwide, perpetual right of access to, and a license to copy, use, distribute, transmit and display the work publicly and to make and distribute derivative works, in any digital medium for any responsible purpose, subject to proper attribution of authorship, as well as the right to make small numbers of printed copies for their personal use.

2. A complete version of the work and all supplementary materials, including a copy of the permission as stated above, in a suitable standard electronic format is deposited immediately upon initial publication in at least one online repository that is supported by an academic institution, scholarly society, government agency, or other well-established organization that seeks to enable open access, unrestricted distribution, interoperability, and long-term archiving. 


\section{The International Context}

There are similar initiatives within LIS, but they differ from E-LIS, in terms of organisation and vision, which in E-LIS is international. It is important to collaborate with these different but similar repositories in order to build a service provider for the LIS context. These repositories differ in their missions, aims and functions. During the short period of existence the organizations that established them found it difficult to sustain them and most of such archives were wound up. When we examined the growth of those archives with strong organizational backing, compared to ELIS it was found to be very slow. The cases of a few sample repositories are discussed below.

E-LIS differs from those models, as it is based on voluntary work of people with different backgrounds and is with a noncommercial orientation. There is neither funding nor interest in receiving a revenue or profit. It is based on the spirit of the open source initiative in computing whereby people from all over the world, work together to build software for the public domain. ELIS works to create a digital library, which is freely available to the LIS community and is managed by an international team of volunteers comprising professionals from many different countries.

\section{National Repositories}

\section{Australia}

\section{Australian Library and Information Association e-prints. \\ http:/ / e-prints.alia.org.au/ launched in (2004). Contains LIS papers from Australia.}

\section{France}

CCSD:@rchiveSIC. http://archivesic.ccsd. cnrs.fr/. Has presently 1485 documents. Server for author self-archiving - of articles and working papers. The majority of the articles are in French. Born from collaborations between numerous French institutions.
CCSD: MémSIC. http://memsic.ccsd. cnrs.fr/. Has 331 documents. Server for archiving - of memories in the field of communication and information sciences (SIC). Most articles are in French and is a collaborative attempt as above.

\section{India}

LDL, Librarians' Digital Library. https:/ /drtc.isibang.ac.in/. Developed by Documentation Research \& Training Centre (DRTC) under Indian Statistical Institute of Bangalore. Is based on the DSpace platform. Contains full text of theses, papers/articles submitted to DRTC. Started with 175 documents.

\section{Namibia}

DSpace at the University of Namibia Library. https://dspace.unam.na:8443/ dspace/Started with a small collection of LIS documents.

\section{Russia}

DSpace at lib.usu.ru. http:// dspace.lib.usu.ru. Launched by Scientific Library of the Ural State University. Is based on the DSpace platform and contains 219 documents in Russian.

\section{Repositories of LIS Schools}

\section{USA}

DLIST, Digital Library for Information Science and Technology. http:// dlist.sir.arizona.edu/. DLIST is the Digital Library of Information Science and Technology, an OA, cross-institutional repository of full-text, electronic resources. The School of Information Resources and Library Science and the Arizona Health Sciences Library at the University of Arizona implemented it. Contains 1541 documents. Accepts documents in English only.

SILS ETDs, University of North Carolina School of Information and Library Science (SILS). http://dc.lib.unc.edu/ $\mathrm{cdm} /$ landingpage/collection/s_papers A repository of SILS student Master's theses (or papers) and doctoral dissertations. SILS 
students are required to submit an electronic version of theses as part of their degree. Contains 2247 ETDs from the School. Uses the DSpace platform.

\section{Local Staff Repositories (Reserved for the Staff of Certain Projects) \\ Italy}

CNR Bologna Research Library EPrints

Service. http://biblio-eprints.bo.cnr.it/ A repository for the self-archiving of research and technical papers in information science, computer science, and related disciplines pertinent to Library activities. It also hosts workshop proceedings and other technical material related to events organised by the CNR Bologna Research Library. Started with a very small collection of 58 items.

\section{USA}

\section{CaltechLIB, Caltech Library System Papers and Publications.}

http://authors.library.caltech.edu/view/ group/Library_System_Papers_and_Pub lications.html

Archive of papers and publications by the professional staff at the Caltech Library System. Uses JITA classification scheme. Contains 47 documents.

Even though the above projects could infuse interest on Open Access subject specific repositories in the regions where the initiatives took place, they lacked organizational set up and policies. Many remained stagnant after a short period of their establishment or became non functional.

\section{E-LIS Continuous Changes and Reforms}

The first decade of existence posed a tough time for the team, which initiated E-LIS. The solutions used required continuous updating and customisations based on experiences and suggestions of editors and users. The system developed into one for providing efficient service. E-LIS gradually evolved into an effective model for selfarchiving of papers. The hosting services and platform got adopted to satisfy the functional contexts and necessities of E-LIS during the last ten years. During this period, which was crucial for sustaining the project, the ELIS server was hosted by AePIC team on machines of the Italian Consorzio Interuniversitario Lombardo per Elaborazione Automatica (CILEA) at Segrate (Milano, Italy). It was a non-profit organization pooling nine Universities in the Lombardia region in Italy. It provides information technology services on behalf of universities and related organizations, public organizations and enterprises. It also provided professional advice for both the planning and the dissemination of advanced technology in the fields of high performance computing, networking services and informatics. A full back up of E-LIS was created weekly and an incremental back up was created every day during the last ten years.

The ELIS Archive is presently supported by AIMS (Agricultural Information Management Standards) the web portal managed by the Food and Agriculture Organization of the United Nations (FAO), International Centre for Research in Information Strategy and Development which addresses open access to scholarly communiation outputs, publishing, preservation of digital information, and standardization, evaluation and visibility of science with special regard to its social aspects; and University of London Computer Centre (ULCC).

The archive was based on GNU EPrints archive software, which was also compliant with the OAI-PMH. It originated from an idea of Steven Harnad's, and was developed at the Electronics and Computer Science Department of Southampton University in the UK by Robert Tansley, Christopher Gutteridge, and Mike Jewell. A GNU EPrints server is an example of an OAI data provider, created for the self-archiving or mediated deposit of electronic resources. Its development has been largely funded by JISC 
(Crow 2004). As a result, EPrints is free, GNU open-source software. E-LIS has a specific discussion list for technicians to discuss technical issues and to find the best solutions for enhancing functionalities and developing new parts to the software that will then be put in the public domain.

ELIS for a short period from 2010 to 2012 changed to DSpace platform. But it found that some features of E-Print software made it more user-friendly and was found apt by the E-LIS family. Most of the staff and users voted for the earlier system after the change from EPrints. So E-LIS again adopted the EPrint platform in which it was born. Hosting was also changed from CILEA to computer Centre of University of London. The lay out and design - graphical aspects were also totally changed in the E-LIS website in December 2012 and was put into effect from January 2013. All these changes were costly in terms of infrastructure and manpower. All the staff members of ELIS are volunteers. So these changes also necessitated the reorganization of the ELIS infrastructure.

Editors from all the countries promoted and popularised E-LIS in their regions and it helped the growth of the collection. All this made E-LIS an effective international subject specific repository model in OA scenario.

A new organizational structure was planned at the end of the year 2012 and was implemented in January 2013. One of the important objectives of the changes is to give an enhanced role for editors. Editors can propose new projects for developing the system and extending the services. Editors are also given the chance to lead approved programmes. They get more visibility in the new system. The duties and responsibilities at each level became clearly defined. But more flexibility compared to that in the past was also provided to encourage more participation and to identify and introduce more changes and innovations.
Several new facilities have been developed in E-LIS after the return to EPrints platform. For example, the layout of the home page and co-related pages have been re-styled and E-LIS has been provided with its own logo. There is also a counter on the home page, which displays the number of papers in the main archive in real time. This development was made possible by a Perl programme written by E-LIS.

\section{The New E-LIS Organizational Model}

Authors who contribute to an e-print archive are participating in a global effort by universities, researchers, libraries, publishers, editors, and readers, to redefine the mechanisms of scholarly communication. ELIS can make LIS research more visible and accessible, which in turn may increase its status and public value.

E-LIS has during its long existence clearly understood the benefits of having a structure, which can incorporate editors from every country. It also got a clear idea of the aspects that worked well, the components that remained still unexplored and those, which needed to be defined more clearly.

One of the important features of E-LIS to be noted is that all its staff members are working on a voluntary basis. So the structure of the system was also formed with the consideration that it will have only a few members who will be continuously active.

E-LIS organizational set-up is built on the following three different layers.

Administrative Board

Executive Board

Editorial Board

A discussion list for each layer provides the basis for action.

\section{Administrative Board}

The Administrative Board (AB) deals with strategic issues including the future direction 
of the initiative, its policies and their impact on the user community, and its connection with other scientific communities. The AB, which deals with policy issues, establishes the E-LIS vision and mission and identifies directives including the operational structure, the choice of hosting and the software to be used. It also looks after editorial coordination, policy concerning copyright and rights issues, and any relationship with external and possible individual partnership.

$\mathrm{AB}$ is the decision-making branch in E-LIS. The AB has about 5 members; the number of members should preferably be an odd number, to enable to take decisions by putting the item to vote if essential. It includes two founder members, a member managing funding and budget, and a member of the hosting organization.

The AB will deal with policy issues, establish E-LIS vision and mission and identify directives including the operative structure, any choice of hosting and software, editorial coordination, policy concerning copyright and rights issues, and any relationship with external and possible individual partnership.

The main tasks of the $\mathrm{AB}$ are:

- To define two year plan of development: objectives;

- To define the budget;

- To outline projects that are defined in the scientific and professional board;

- To appoint members to the scientific and professional board;

- To identify areas for improvement within the OA international framework by increasing the visibility of E-LIS; and

- To promote and enhance the relationship with external partners.

\section{Executive Board}

Executive Board (EB) serves as the executive branch of E-LIS. Its members vary in number, depending on the design lines of development. The $\mathrm{AB}$ appoints the members. The EB deals with the professional and technical services, not only related to technical management, but also from a scientific point of view and in compliance with the managerial guidelines. The EB also ensures consistency on graphical and informative web pages attached to ELIS. The EB works in two parallel lines:

- Long-term lines of development following the E-LIS vision and mission, assured by three specific coordinators;

- Implementing project guidelines which, when implemented and tested, become an integral part of the main activities of E-LIS.

EB looks after the development and implementation of software and its enhancement or value addition for required functionality and all matter concerning OAI framework. It is also concerned with over all searching structure and methods and procedures for harvesting.

The main tasks of the EB are the following:

- To contribute to the management of ELIS and its technical and professional development;

- To establish the guidelines of the project identified by the administrative board in relation to the objectives of the Development Plan; and

- To report the evolution of individual project guidelines to the $\mathrm{AB}$.

\section{Editorial Board}

Editorial Board (EDB) is responsible for the approval/rejection of documents in E-LIS. The EDB is managed by a coordinator with the support of a group of regional editors. The EDB helps the growth of collection in E-LIS and maintains the quality of the content, ensuring accuracy of metadata, and formats in accordance with the rules, policies and standards set. The main tasks of EDB are the following. 
- To maintain and manage metadata at country level, approving the work arriving in the buffer and contacting authors and / or applicant in cases of doubt;

- To support the promotion of E-LIS in his/her own country and/or in their area of proficiency;

- To organize events for the promotion of E-LIS and/or writing articles in trade magazines;

- To contact publishers for matters relating to the filing of articles in magazines (in consultation with the Copyright and rights management coordinator in cases of copyright policy if necessary).

\section{Structure of E-LIS}

Users perceive two main structures around which all the contents are organised; both structures have two levels. The first structure is the classification scheme for LIS (called JITA) devised by E-LIS. In JITA the subject of Library and Information Science is first divided into main subject divisions and subdivisions. The second is a geographical ordering based on continent and country. Users can navigate these structures to retrieve the required information. They can also view documents by browsing by author or year. Information can also be accessed via the search interface through a basic or an advanced search. In order to improve the search mechanisms, E-LIS has enhanced the full-text search furnished by the EPrints software it uses.

One who submits a document is required to assign subjects and keywords from the JITA Classification Scheme. JITA has a second-level, browse-by-country view, which has over 120 sub-sections. This simple scheme, which has also been adopted by other repositories, is the result of the fusion and re-arrangement of the News Agent Topic classification scheme and the Review of Information Science (RIS) classification scheme (JITA, 2012). The JITA classification scheme is not intended to be a comprehensive classification scheme, but to facilitate document retrieval through the archive's browsing facility. It is divided into twelve blocks (categorised alphabetically from A-L), which have been created around the three following implicit (virtual) areas:

- Theory and generalities (general level): This is divided into: theoretical and

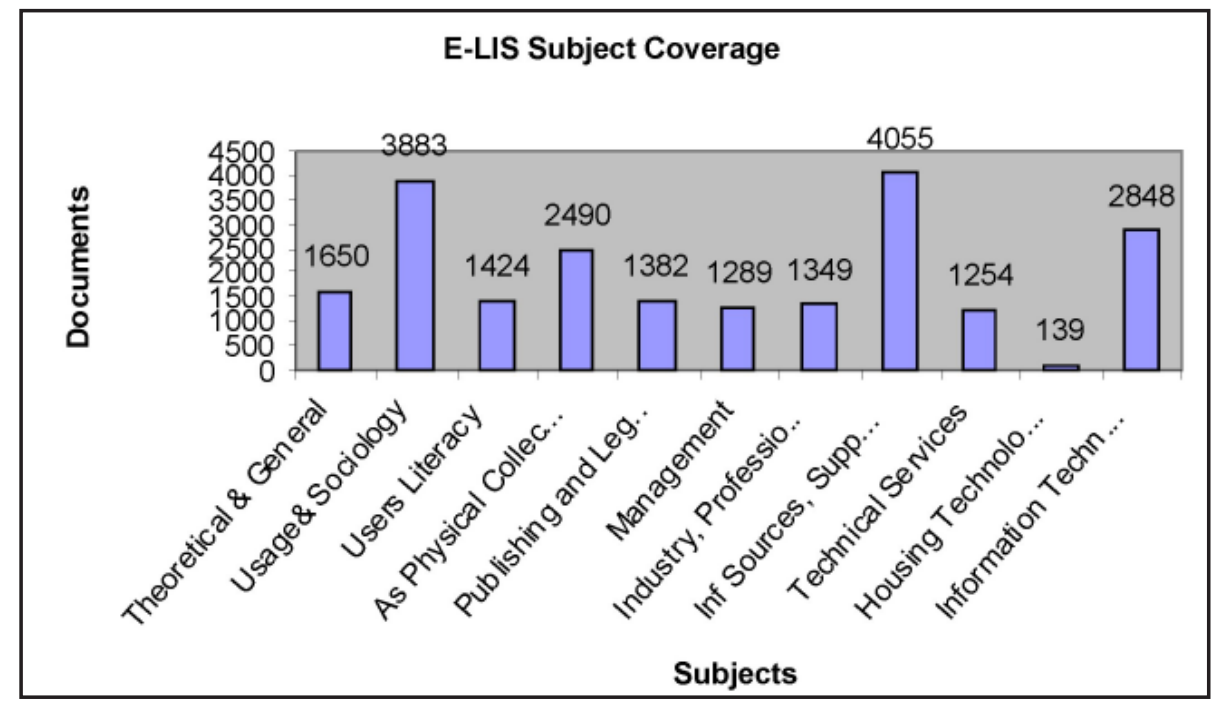

Figure-2: Subject Coverage of ELIS under Main Sections of JITA 
general aspects of libraries and information; information use and the sociology of information.

- User-oriented, directional, and management functions (intermediate level): Socio-economic and legal issues are included here. This divides into: users, literacy and reading; libraries and information repositories; publishing and legal issues; management; industry, profession and education.

- Objects, pragmatic issues and technicalities (on a specific level): This covers information sources, supports and channels; information treatment for information services; technical services in libraries, archives and museums; housing technologies; information technology and library technology.

In ELIS it is possible also to browse by country. This gives a truly international aspect to the archive and is particularly aligned with the organisation of the EB whereby work is channelled through international staff on an individual country basis.

\section{Strategic Issues}

The core of the organisational model is the Administrative Board. It has the responsibility for determining the policies and procedures at international level and forming the future vision of the archive. It guides development of E-LIS, keeping in mind the national and international needs. In some countries, library and information service providers want to create national archives for LIS instead of uploading papers into an established international archive, such as E-LIS. This is understandable because there are many technical difficulties associated with the use of different languages, alphabets, and non-alphabetic languages and with the consequent problems of input, output and the sorting of data and author names. E-LIS co-operates with each country individually to decide the best solution to solve the technical and non-technical issues to ensure international visibility, whilst national interests are also served. One idea, currently being debated, is to create a service provider with a harvester to gather national metadata from the national LIS archives, which could become part of the RCLIS infrastructure.

Thirty countries are currently involved with E-LIS and each of them works towards a common vision while maintaining their own cultural individuality. The editors are entrusted with the responsibility of promoting E-LIS within their own country and every editor has a different approach, which is tailored to the specific needs of that country. The status of library and information service profession, which differs significantly from country to country, is the result of several factors such as the role of the professional association or of the LIS School, institutional assets, initiative on the digital library front, the impact of LIS in the social environment and how much OA has become a part of scholarly communication. However, all the editors share a common vision, and bring their own disciplinary and personal experience to bear on the position.

\section{Country wise Coverage}

In E-LIS, as mentioned earlier papers can be viewed on an individual country basis. It can in addition to enabling information retrieval efficiency promote LIS research of local relevance in concerned regions. A continent wise analysis of papers archived in ELIS is presented in Figure 2. In the LIS community most published papers traditionally came from the US, UK and Europe, but in ELIS, where OA encourages a wider audience, it has been noted that high quality papers now come not only from the countries believed most innovative, but also from developing countries, such as India, and others in Africa, Asia, Eastern Europe, the Russian States and also Central and South America. This has been a very welcome, if unforeseen, development. The sense of this inclusiveness is highlighted by the recent collaboration with Cuban librarians, who 


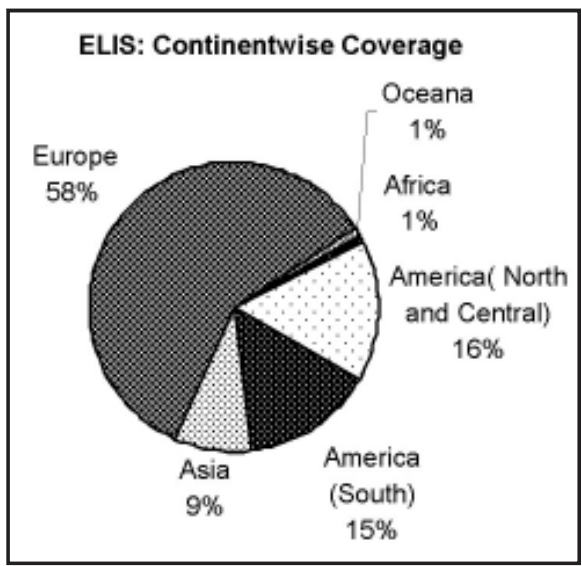

Figure 2: Continent wise Representation in Collections of ELIS

have deposited papers in E-LIS from ACIMED, the Journal of Information Professionals in Health Science institutions.
The Open Access vision of E-LIS can be juxtaposed against the blockade imposed by the United States on Cuba, which is designed to prevent the publication of Cuban papers in the main U.S. journals. The Cuban case illustrates that E-LIS knows no borders, and negotiations are in progress regarding the involvement of Arabic countries, Israel and China. Collaboration with other countries has been very beneficial for E-LIS. In particular, Indian librarians have demonstrated a very high competency in bibliometric issues, which has proved very stimulating for the E-LIS community as a whole.

In E-LIS about $9 \%$ of contributions is from Asia. Among Asian countries Indian Community is very active. About 700 papers

\section{$e$ - LiS \\ e-prints in library \& information science}

$\Delta$ Up a lexal

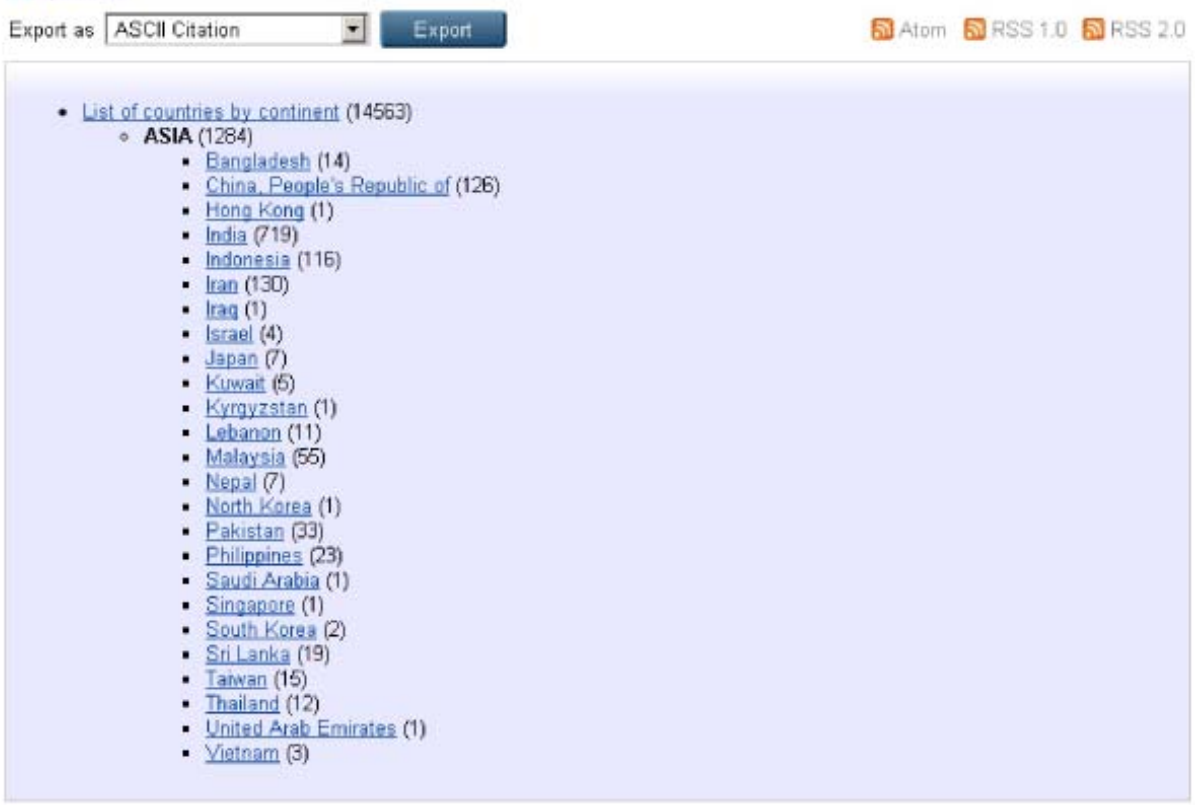

Number of items at this level: 0

Fig. 3: Country wise Browsing: Papers From Asia 
in E-LIS are deposited from India (Figure3). This consists of papers in the languages of English, Hindi, Kannada Malayalam and Marathi. The contribution of Indian depositors has made an impact on other communities also.

E-LIS collaboration with India started in 2004. Venkatrao Lakshmanrao Kalyane was the first editor for India who worked from 2004 to 2006 . He worked very hard for the whole LIS community. His service has helped E-LIS to extend its services to most regions in South Asia. He published more than 100 papers on scientometrics and of them 74 items are deposited in E-LIS. He spread the message of E-LIS in India till he passed away in 2010.

\section{E-LIS Policies}

E-LIS is driven and directed by its policies, which determine its identity and quality. Merely putting software on a machine is not sufficient to create an archive. In the case of an archive like E-LIS; its organisational model is the sum of its policies. An archive without policies is like a ship sailing in the sea without a navigator. The principal policy concerns of E-LIS, which are discussed and democratically agreed upon by the editorial staff, are the following:

Mission: Its aims and objectives, what it is and where it is going, its target audience and the communities involved. Its international mission, (previously discussed) is clearly outlined on its website.

Submission Policies: Who can deposit material and how such deposits should be made (described in the following section).

Copyright Policies: They (described below) are fundamental to any repository and are assigned due importance in E-LIS.

Organisational Model: Which (already described above) is the core of any repository and determines its institutional or disciplinary nature.

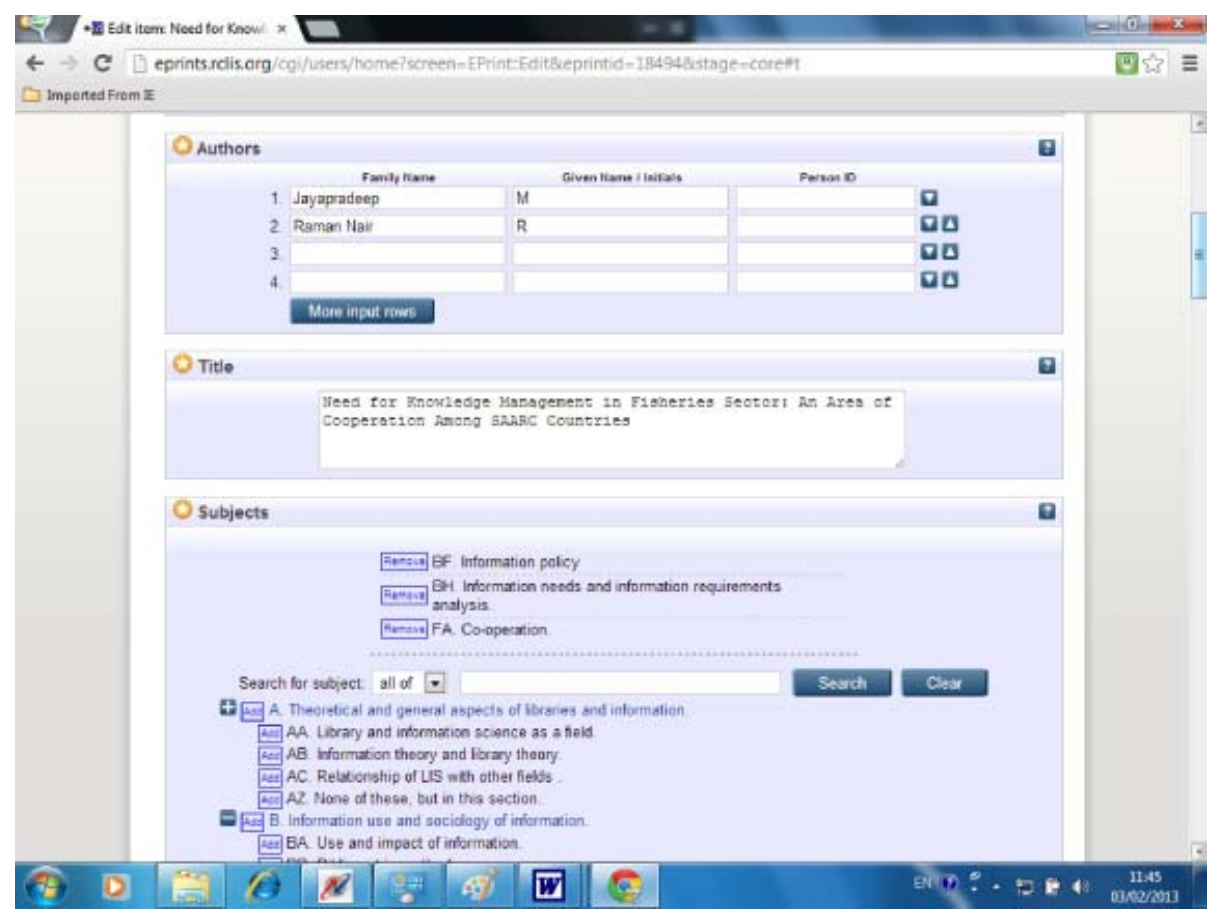

Figure 4: E-LIS Submission Interface 


\section{Submission Policy}

Every library and information science professional engaged in research, teaching or practising anywhere in the world can deposit papers in E-LIS as it promotes intellectual freedom and maximisation of impact in the LIS discipline. With E-LIS, as with arXiv in the physics field, experience has shown that this freedom has not led to the submission of low quality papers because authors are aware that a very wide community of peers will ultimately judge their papers on a virtual basis. The technical submission requirement is that authors who wish to submit a document must register in order to obtain a user ID, which is also the basis for obtaining author-view browsing. Librarians, libraries, research institutes, organizations, and individual researchers involved in LIS and related fields are encouraged to make use of, and contribute to, the expansion of the archive and in turn to the critical mass of information available and useful for the building of digital libraries.

The E-LIS submission policy states that the archive accepts any scientific or technical document, published or unpublished, on librarianship, information science and technology or related activities. In this context, categories for different types of materials have been created with respective sets of metadata. The criteria for acceptance are that the e-prints are relevant to research in LIS fields, and that they have the form of a finished document ready to be entered into a process of communication. Publications may include preprints, post prints, conference papers, conference posters, presentations, books, book chapters, technical reports/departmental working papers, theses, and newspaper and magazine articles.

Submitted documents are placed in the submission buffer for approval or rejection by E-LIS staff. Rejection is on the basis of pertinence to the archive. E-prints may also be returned to the author for metadata modification or if there are problems with the electronic file or format. Editors may make formal corrections but they do not make substantial modifications to the text or contents of the registered data. Documents in the submission buffer are manually reviewed to ensure that they conform to E-LIS policy. E-LIS staff controls the metadata quality of the document and are allowed to make changes if the metadata is incorrect. Generally, a paper becomes publicly accessible in two working days after its deposit in E-LIS. The editor from the country of the depositor conducts the approval process. Once submitted, a document cannot be removed from the repository. Even when changing institution, Stevan Harnad argues that 'Wanting to remove one's work from the [repository of the] old institution is [as] absurd as wanting to remove it from the shelves of one's old library - or any library'. Indeed, removal would seriously hamper the communication process and users would be unable to chart the evolution of the idea or debate. Furthermore, because documents remain in the archive for posterity, the submission of inferior work is thus discouraged (Harnad, 2004).

As an international open archive, E-LIS supports all languages; however, if a document is in a language other than English, it must include English abstract and English keywords. If the English abstract is missing, the editor inserts it on behalf of the author. The document formats accepted are; PDF, PostScript, Tex, LaTeX (DVI), HTML, XML, ASCII (text), PowerPoint, MS Word DOC and RTF. The use of HTML and PDF formats are strongly recommended.

\section{Copyright Policies}

E-LIS does not want to infringe copyright. $\mathrm{OA}$ is encouraged where possible, but authors can restrict access to their papers if necessary. Access can also be restricted to the group of registered users of the E-LIS 
archive, which is a limited and known group of people. Furthermore, access can, in special cases, be restricted to only the depositor and archive staff like the archive administrator and any selected editors, evaluators, etc. in particular countries and organizations.

All works residing on the E-LIS server remains the property of the author. The author holds the copyright for the prerefereed preprint and therefore, it can be selfarchived without any other permission being sought. An author's works are that author's own intellectual property and they therefore own copyright and other proprietary rights until and if they grant otherwise. Authors submitting their works to the repository are responsible for ensuring that the documents they archive do not have any restrictions on their electronic distribution imposed by a third party (such as a publisher). A prerefereed preprint can be self-archived at a time when no copyright transfer agreement exists and so the author holds exclusive and full copyright; the author may no longer have the right to self-archive a refereed post print if a copyright transfer agreement has been signed granting all rights to the publisher. In general, when an article is published in a journal, copyright is transferred to the publisher. Most journals permit selfarchiving of the preprint and sometimes also the post print, but it depends on the publisher's copyright policy. To avoid infringing any copyright, authors can deposit a post print inside the archive with restricted access. Another way round this problem is for the author to request that the publisher allow them to retain certain rights, e.g. the right to deposit the post print in an open access archive, or to place a copy on their homepage. Some publishers have stated that they grant these rights as a standard procedure. Alternatively, authors can replace the full text of the preprint with a link to the published version, if it is freely accessible. Copyright law gives to the creator of a work exclusive right, which may be both segmented and transferred to others.
Publishers have adopted various policies to facilitate author self-archiving. To help authors and editorial staff establish the copyright situation for deposit of a particular item, E-LIS uses the SHERPA database of publisher copyright policies and selfarchiving. It is a programme which supports projects aiming to achieve the 'disclosure of institutional assets' with the vision of setting up a 'web of resources built by groups with a long term stake in the future of those resources, but made available through service providers to the whole community of learning.' It investigates key issues in creating, populating and maintaining e-print collections, including specifically: Intellectual Property Rights (IPR), quality control, collection development policies, business models, scholarly communication cultures, and institutional strategies (SHERPA, 2012). In addition, E-LIS is aligned with the Rights Metadata for Open Archiving (RoMEO) project, which cites the E-LIS policy on metadata. E-LIS's metadata policy permits third parties to collect metadata from the archive via mechanisms that create end-user services to support the discovery and presentation of the archive content (ELIS, 2012). The general policy is to allow harvesting of metadata but not the harvesting of full content.

Whatever the policy, the authoritative document for a published paper is the copyright agreement signed with the publisher. Fortunately, many publishers are adapting to the changing environment of electronic publishing. Nevertheless, some publishing policies contain ambiguities or have different approaches to self-archiving. For example, Elsevier, which publishes journals such as 'International Information and Library Review' and 'Library and Information Science Research', has some restrictions regarding self-archiving. Its new policy appears friendly to self-archiving, but some important restrictions are evident in the details, for example, the conditions regarding deposits are as follows: 
- Deposits must be on author's personal or author's institution's server;

- Published source must be acknowledged;

- Deposits must link to journal home page;

- Publisher's version/PDF cannot be used.

E-LIS can agree with the last three conditions, but operates in contradiction with the first condition, which disallows deposit in a disciplinary repository such as E-LIS. According to Elsevier policy, therefore, authors can only deposit in two places:

a) on the author's personal web page. There is no co-ordination to facilitate the search and retrieval of scientific papers posted in the vast ocean of personal web pages.

b) on the institutional server. Institutional servers are not well developed or are nonexistent in many universities throughout the world. The current reality is that there are very few institutional servers and their archives are not well stocked.

Elsevier's decision to only allow self-archiving of preprints on personal or institutional servers deprives the author of the possibility of archiving in well-established, well-run, disciplinary repositories with good reputations. It will prevent the exposure the paper would have got in an Open Archives System. For many authors, a disciplinary archive is more relevant than an institutional one, particularly as many would prefer to display their work and search for papers on a disciplinary, rather than an institutional, basis.

In an OA repository such as E-LIS, a user may find an exact copy of a paper from a journal, which is normally sold by a subscription. When a publisher's archiving policy is quite open it is possible to place a version (preprint or post print) of a published paper in a repository. A user who knows how to use repositories can find high quality material in a free-access database, instead of acquiring it via a payment.
Occasionally, a paper can be found in an aggregator site through a pay-per-view model (on average at $\$ 30$ per article), but in some cases papers are only found in electronic journals, some of which are 'open access' and some 'toll access'. Some very high quality papers published in toll access LIS journals are available freely within an open access repository but it is imperative that archiving authors have a clear understanding of the different archiving policies adopted by publishers.

\section{E-LIS Editors}

All work performed by the editorial section is developed by the editorial staff from discussion on a mailing list. Topics include metadata issues, guidelines for cataloguing, promotion of E-LIS and OA in general, and questions which arise from international cooperation. The principal editorial aim is to reflect the best practices of library and information service profession in each country by inviting the top scholars in the discipline to contribute to E-LIS. Therefore, the choice of editor for a particular country is crucial as they must be thoroughly conversant with the debates and personalities involved in the LIS disciplines in their country and they must also have the dynamism to promote E-LIS and the commitment, talent and patience for organising people, events and documents. Again, it must be stressed that this activity is currently done on an entirely voluntary basis, but it is evident that it has been of immense benefit to the LIS discipline as a whole. International cooperation can facilitate debate on current issues at many levels and provide the editors, on a personal level, with new professional experiences and expertise. Editorial tasks include:

- Creating and maintaining contact with university-based LIS academics and researchers and LIS-related course personnel;

- Approaching LIS publishers, with a view to obtaining permission to add articles 
from journals, both those already freely available on the web and those still using authentication systems to E-LIS;

- Sending publicity e-mails to national mailing lists and submitting press releases to web-based publications;

- Writing literature reviews regarding Open Archive activities and writing journal articles on the E-LIS initiative.

\section{Metadata Quality}

Fundamental to obtaining high metadata quality are the repository's 'guidelines for cataloguing', which were created to facilitate editorial work and standardise metadata. The purpose of having the guidelines is to ensure that E-LIS adopts recognised, established practices for the creation of records. These guidelines assist the editors in assigning the correct value for each metadata field and were created taking into account the following points:
- Types of documents accepted

- Variety of contents accepted

- Who is submitting the material

- Metadata standards

- Advice concerning file formats and preservation of documents.

Each of the twenty-three document types have their own set of metadata, which is checked in accordance with the editorial guidelines set by the international editorial committee. The twenty-three document types are:

- Preprint

- Thesis

- Book

- Book Chapter

- Bibliography

- Guide/Manual

- Tutorial

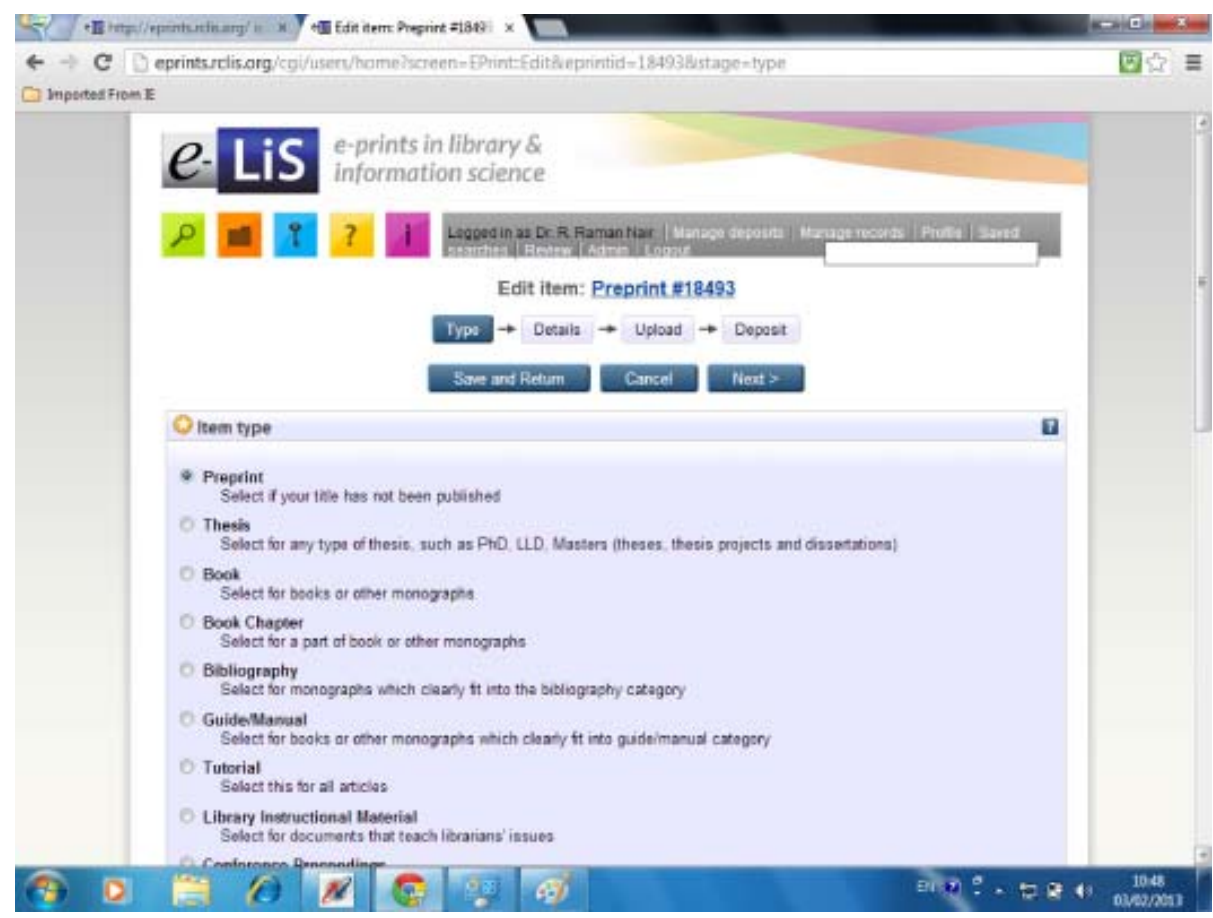

Figure 5: E-LIS Interface for Defining the Type of Document. 


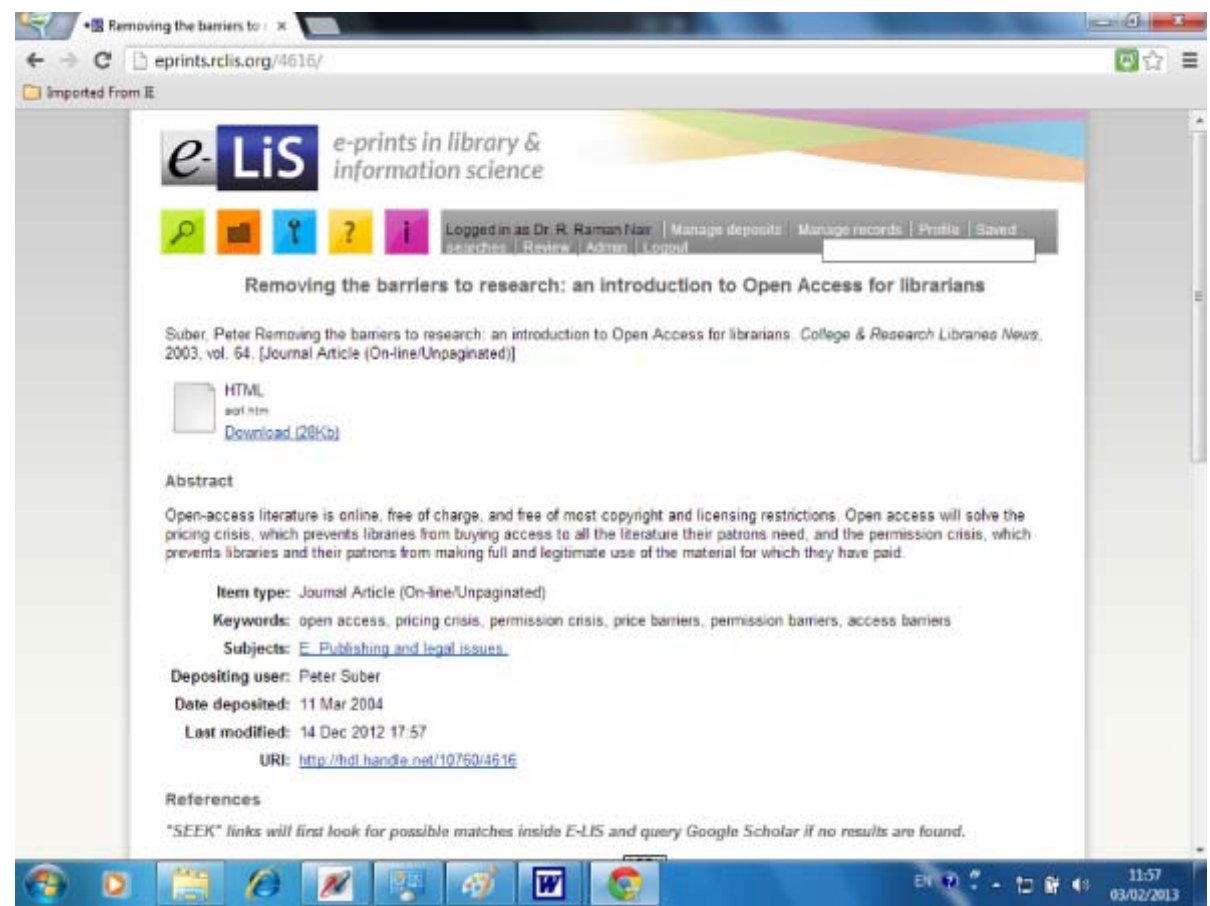

Figure 6: Display of all Metadata Fields and Labels of an Item.

- Library Instructional Material

- Conference Proceedings

- Conference Paper

- Conference Poster

- Presentation

- Project/Business Plan

- Report

- Departmental Technical Report

- Technical Report

- Journal Article (On-line/Unpaginated)

- Journal Article (Print/Paginated)

- Review

- Newspaper/Magazine Article

- In Collection

- Dataset

- Other

In addition to a common metadata core (Dublin Core) there is also a specific configuration of metadata for each category of these document types.
Furthermore, as E-LIS is a tool for librarians, a function - 'show all fields' - has been created which allows users to view an item's full list of metadata with field labels.

\section{OAI Protocol Compliance}

Access to information is essential in a democratic society and the OAI framework helps to furnish tools and standards, which give full access to information. Public health, the economy and public policy all depend on access to, and use of, information including copyrighted works. Technical solutions based on Open Source software, such as those adopted by E-LIS, are recommended to build open digital libraries. The OAI develops and promotes interoperability standards that aim to facilitate the efficient dissemination of content. The OAI Protocol for Metadata Harvesting (OAI-PMH) allows third-party services to gather standardized metadata from repositories and conduct searches amongst that assembled metadata to identify 
and ultimately retrieve documents. E-LIS is part of such a framework because the archive was built around OAI standards allowing the submitted records to be shared with, and contribute to, a growing global network of distributed, interoperable, institutional archives. Since the beginning of 2003 , E-LIS has been validated within the OAI framework and has been registered in the official OAI register of data providers as an OA archive. All the metadata present in ELIS is therefore exposed to harvesters, one of which is OAIster, the OAI service provider which harvests from all OAI archives.

\section{Access to Content in E-LIS}

The E-LIS service is accessible in two ways. First, a search engine can be used to search across the bibliographic descriptions (metadata). Two search screens are available for simple or advanced searching. The simple and advanced searches can be conducted within the full-text of documents. The search can be one or more of the attributes of the document like title, creators, words in the abstract, date, keywords, subjects, item type (preprint, thesis, book, book chapter, bibliography, guide/manual, tutorial, library instructional material, conference proceedings, conference paper, conference poster, presentation, project/business plan, report, departmental technical report, technical report, journal article (on-line/ unpaginated), journal article (print/ paginated), review, newspaper/magazine article, in collection, dataset etc), department, editors, status (published, in press, submitted, unpublished), refereed publication, type (text, slideshow, image, video, audio, archive etc. of the documents).

Second way is, documents are offered for browsing by author/editor subject, country, year, journal/book/and conference. Browsing by Author takes users from alphabetical list of authors to their collections. Subject browsing provides for search under JITA Classification Scheme' main and sub heads. Country search enables search under continents and country. Items deposited in the previous week can be viewed within the Latest Additions section linked from the home page. Having browsed or searched the archive, users can then view the metadata associated with a document, and download a copy of the paper if the access is not restricted.

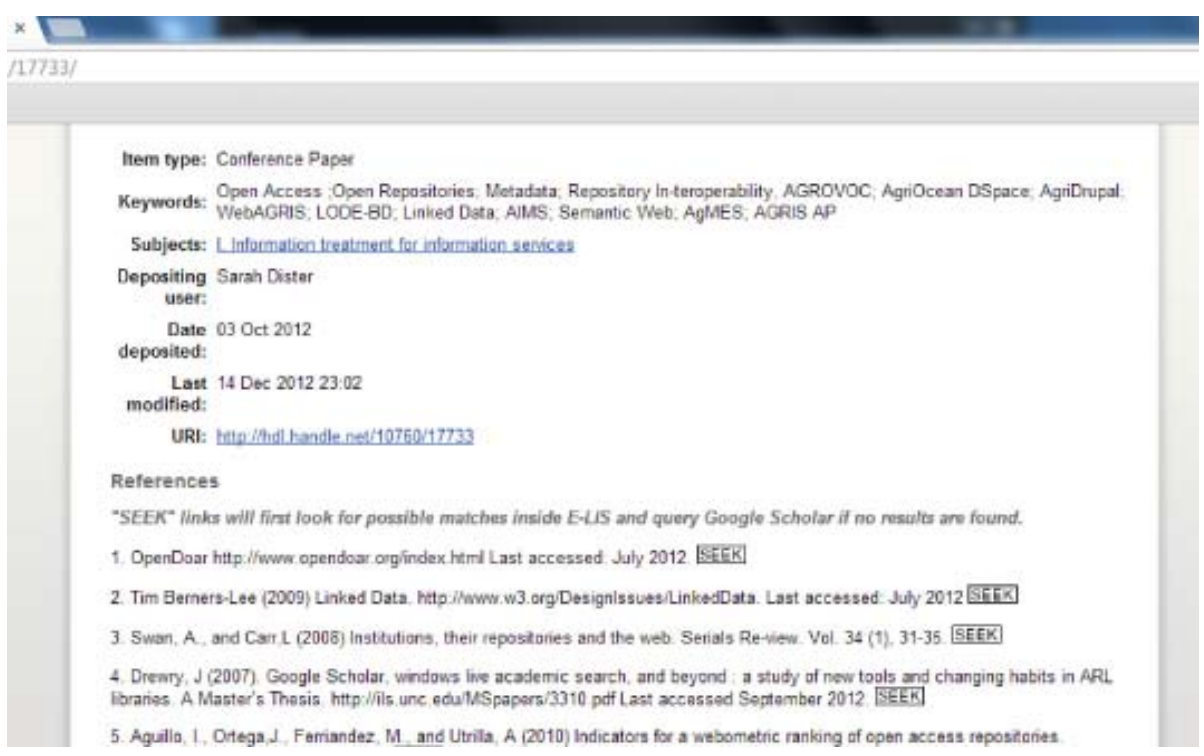

Figure 7: Para Tools to Extract Metadata from Citations and Link the Item on the Web 


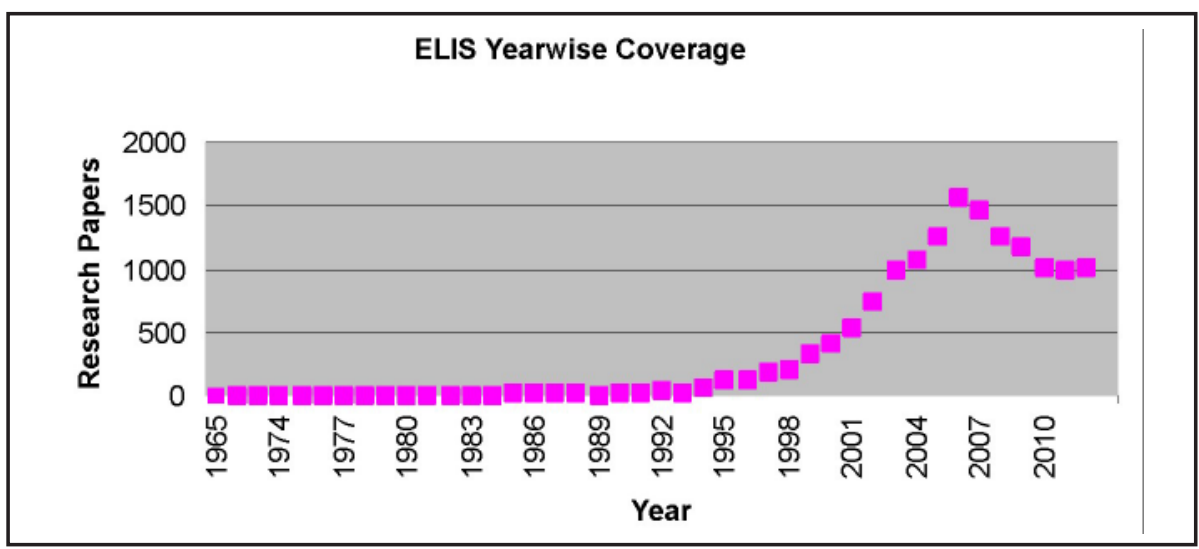

Figure 8: Year wise Coverage of ELIS Collection

\section{Reference Linking}

E-LIS uses Para Tools for reference linking, which is a set of Perl modules for parsing citations (the references at the end of papers). E-LIS has installed an enhanced version of Para Tools so that citations point directly to documents present on the Web and an indication is given if the cited document is itself in the E-LIS archive. Para Tools' main library uses a template-matching technique to extract metadata from citations, while secondary libraries provide support for Open URLs and reference extraction from documents. Its main aim is to perform accurate citation parsing and there are specific modules within E-LIS to support this function. Another module can take parsed metadata and create/enrich Open URLs and there is experimental support for extracting references from files (including PDF, PostScript and HTML). Other modules will be added as new ideas are proposed.

\section{Period wise Coverage of Publications}

The earliest paper available in the ELIS archive pertains to the year 1965. For the year 1995 there are 115 documents. The highest number of records are for the year 2006. For the later years the number of records added went on diminishing. The decline became steep during the period of platform changes to DSpace and then back to EPrints. Now the system has become steady and the annual growth has regained its speed.

\section{Conclusion}

The challenges and opportunities created by a vision of a global collection of papers in the LIS field gives E-LIS the impetus and motivation to stimulate participation in the venture and to further develop its support for international research activities. The promotion of E-LIS also enhances importance of the OA movement in general as it is one of the few biggest subject specific OA collections existing in the world and ensures quality of research in the field in numerous ways. So E-LIS can be regarded as a tool for the dissemination of the $\mathrm{OA}$ philosophy. In terms of strategic issues as the ones listed below E-LIS can serve as a good guide for other OA projects:

- Reporting statistics on authors and papers;

- Adding Creative Commons licences to metadata in the archive;

- Involving other subject specific/LIS partners by wide promotion in every country;

- Funding and long term sustainability;

- Continuous updating of technical functionalities;

- Enriching metadata on the basis of the 
AMF (Academic Metadata Format) model;

- Building of Service Provider from within the subject field.

E-LIS members believe that authors need to have appropriate tools and ways to maximise the impact of their research papers in order to improve scholarly communication. The production of reliable and relevant statistics structured on the basis of author output and single papers is an important and strategic issue. The development of tools to do this will play a leading role within the national frameworks in respect of evaluation of research channels, and to develop a common model where all intellectual output can find wide visibility and easy dissemination among different scholarly communities at the global level. Therefore, the maximisation of author visibility in the LIS field is a priority for E-LIS. E-LIS wants to keep its focus on this aspect, pursue efforts collaboration so that funding for the development of new software can be easily obtained (De Robbio, 2003). The long-term sustainability of E-LIS is a matter of great importance, strongly connected to its partnership and future alliances.

As previously stated, E-LIS is engaged in several fronts. Its future development will focus on the creation of a service provider tool. METALIS (Tajoli, 2005), a prototype service provider for the LIS field has been developed which now harvests metadata from nine repositories that offer full-text papers and documents about library and information science. One possible option is to combine the advantages of using centralised archiving with a distributed system composed of national repositories for each participating country. The creation of a distributed network archive, within an RCLIS infrastructure, could be subject-based and would involve combining several archives. In a model such as this, information would always be retrievable, no matter where it was deposited.
The possibilities offered by Creative Commons (CC) are of great interest, particularly Science Commons, an exploratory project to apply the philosophies and activities of Creative Commons in the realm of science, the mission of which is to encourage scientific innovation by making it easier for scientists, universities, and industries to use literature, data, and other scientific intellectual property and to share their knowledge with others. Creative Commons works within current copyright and patent law promoting legal and technical mechanisms that remove barriers to sharing knowledge (Creative Commons, 2012). Financial or human resources need to be found in developing software that can be added to the EPrints software, which will manage $\mathrm{CC}$ licences attached to the metadata submitted by authors during the selfarchiving process. These licenses will not release the works under it into the public domain, but they will encourage creative reuse of works in ways that full copyright protection does not. Licenses help authors retain their own copyright while allowing certain exceptions in certain conditions. The CC lawyers have ensured that licences are enforceable in as many jurisdictions as possible. Furthermore, the practices of the RoMEO project regarding metadata were considered when developing the set of rights expressions under CC to meet the requirements of academic research papers. The licences have three incarnations: a simple 'human-readable' version, a 'lawyer-readable' licence document, and 'machine-readable' rights metadata.

As the RoMEO project decided on the use of CC licences to express rights over academics' open access research papers and metadata, E-LIS will follow suit. The joint OAI/RoMEO Technical Committee is working to create opportune guidelines and is also exploring a methodology for disclosure and harvesting the rights expressions inside metadata via the OAI- 
PMH. OAI/RoMEO suggests a fivepronged approach given below :

- Default, repository-wide rights expressions covering the metadata;

- Default, repository-wide rights expressions covering the resources;

- Optional, set-level rights expressions covering the resources;

- Rights expressions covering the individual metadata records;

- Rights expressions covering the individual resources.

E-LIS needs to analyse how it should adopt the correct metadata and CC licences, at which level of granularity the new metadata rights management procedure should be implemented, and at which step in the selfarchiving process it should be introduced.

The final point to denote is the issue of enriching the metadata. The relationship between the OAI protocol and Dublin Core (DC) was discussed during a breakout session co-ordinated by Carl Lagos during the CERN Workshop Series on Innovations in Scholarly Communication: Implementing the benefits of OAI (OAI3) (CERN, 2004). Since the first release of the OAI-PMH, unqualified Dublin Core (DC) has been the basic metadata format, useful for interoperability. There was discussion on the quality of metadata being created by nonqualified people during the self-archiving process. With regard to the question of using other metadata formats, it was suggested that the mandatory link to DC be kept, but that the DC type and identifier should be used and then the record linked to the richer metadata format. It is imperative that any solution should not invalidate previous work, particularly because the Dublin Core is intended mainly for resource discovery of 'document-like objects', and OAI-PMH is being applied in a number of other contexts. DC is useful not only for indexing, but also for listing and browsing results. RCLIS is working to provide information to LIS communities about valuation and perhaps changing this requirement, which may interfere with application of the protocol. Therefore, while maintaining Dublin Core as mandatory, the use of the richer formats must be considered in parallel within the RDF framework like the following:

- MARC coming from traditional library environments;

- LOM for learning objects placed inside e-learning platforms or learning object repositories;

- OLAC the Open Language Archives Community metadata set based on qualified Dublin Core, but allowing the use of extensions to express communityspecific qualifiers;

- AMF - Academic Metadata Format: a particular format to support scholarly communication developed by Thomas Rachel and Simeon Warner (Rachel, 2001).

An alternative to E-LIS could be to consider Dublin Core by default, and to use other rich metadata, such as the AMF, more tailored to scholarly communication needs (Imma 2004). The AMF format is associated with a conceptual framework that comprises four classes of entities: resources, groups of resources, people and institutions.

The entirely voluntary character of E-LIS must again be stressed, as it gives a particular connotation to this support system which facilitates co-operation, promotes consensus, facilitates forms of expression and identities of OAI group members, and nurtures relationships between different actors and countries in this wide, international community. E-LIS is confident of its progressive and comprehensive nature in technical matters and content, and particularly in the global nature of its vision and philosophy, and in its attempt to enhance and improve professionalism within the discipline. However, it is not devoid of challenges, particularly concerning its budget 
and the resources needed for a more incisive performance, and as grants and other sources of funding are elusive, the success of E-LIS is largely based on the earnest efforts of its members.

\section{References and Further Reading}

CERN (2004). CERN Workshop Series on Innovations in Scholarly Communication: Implementing the benefits of OAI (OAI3). 12-14 February 2004. Retrieved: May 22, 2013.

Agenda: http://agenda.cern.ch/ fullAgenda.php?ida $=a 035925$. (URL of workshop: http://cern.ch/oai3/)

Creative Commons (2012). Creative

Commons. Retrieved: May 22, 2013. http:// creativecommons.org

Crow, Ray (2004). A Guide to Institutional Repository Software. Open Society Institute. Retrieved: May 22, 2013. http:// www.opensocietyfoundations.org/ openaccess/resources/guide-to-institutionalrepository-software/

De Robbio, Antonella (2003) E-LIS: un Open Archive in Library and Information Science. Bibliotime VI (1) (Italy) Retrieved: May 22, 2013. http://eprints.rclis.org/5366/ 1/aib-notizie-ELIS.pdf

De Robbio, Antonella (2003) E-LIS: un open archive per library and informations science. AIB Notizie 15(2), 12. (Italy). Retrieved: May 22, 2013. http://eprints.rclis.org/5366/ 1/aib-notizie-ELIS.pdf

ELIS (2012). E-LIS Copyright Policy.

Retrieved: May 22, 2013. http:// eprints.rclis.org/policies.html

Harnad, Steven (2001). The self-archiving initiative: freeing the refereed research literature online. Nature 410 (26 April 2001), 1024 - 1025 . Retrieved: December 22, 2012. http://www.ecs.soton.ac.uk/ harnad/Tp/ nature $4 . h$ tm

Harnad, Stevan (2004). Central versus institutional self-archiving. American Scientist Open Access Forum. Posted Tue Nov 022004. Retrieved: May 22, 2013. http:// www.ecs.soton.ac.uk/ harnad/Hypermail/ Amsci/4112.html
Imma, Subirats Coll and Barrueco Cruz, José Manuel (2004). Un archivo abierto en ciencias de la documentacin e informacin. El Professional de la Informacin 13(5), 346-352 .

Retrieved: May 22, 2013. http:// eprints.rclis.org/5578/1/barcelona.pdf

Imma, Subirats Coll, and Arencibia Jorge, Ricardo and De Robbio, Antonella (2004). Eprints for Library and Information Science (E-LIS) : la tecnología al servicio de la bibliotecología y las ciencias de la informacin. ACIMED 12(6). (Cuba). Retrieved: May 22, 2013. http:/ / eprints.rclis.org/5776/1/ aci07604.pdf

JITA (2012). JITA Classification Schema of Library and Information Science. . Retrieved: May 22, 2013. http://aims.fao.org/kos/jitaclassification-system-library-and-informationscience

Kumar, Anil and Kalyane, V. L. (2004) Bibliographics for the 983 EPrints in the live archives of E-LIS : trends and status report up to 7 th July 2004, based on author-selfarchiving metadata. Retrieved: May 22, 2013. http://eprints.rclis.org/5212/1/July2004.pdf

Lewandowski, Dirk (2004) E-LIS : E-prints in Library and Information Science.

Proceedings Internationales Symposium fur Information swissenschaft (ISI). Retrieved: May 22, 2013.

URL: http://eprints.rclis.org/archive/ 00002532/

Medeiros, Norm (2004) A repository of our own: the E-LIS e-prints archive. OCLC Systems \& Services. 20 (2), 58-60. Retrieved: May 22, 2013. http://eprints.rclis.org/5001/ 1/ELIS_OTDCF_v20no2.PDF

OAPC (2003). Definition of Open Access Publication. [Drafted during the Open Access Publishing Conference (OAPC), April 11, 2003] Open Access Now : Bethesda Principles. Retrieved: May 22, 2013. http:// legacy.earlham.edu/ peters/fos/ bethesda.htm

Rachel, Thomas and Warner, Simeon M (2001). A metadata framework to support scholarly communication. International Conference on Dublin Core and Metadata 
Applications (DC-2001) October 22-26, 2001 (National Institute of Informatics, Tokyo, Japan, 2001). Retrieved: May 22, 2013. http://openlib.org/home/krichel/papers/ kanda.html

Raman Nair, R (2010). Open Access Initiatives and ELIS Archive in Library and Information Science. Challenges and Changes in Librarianship: Sewa Singh Festschrift volume. New Delhi: BR, 29-51. Retrieved: May 22, 2013. http:/ / eprints.rclis.org/13301/1/ 2010Sewa.pdf

RCLIS (2012). RCLIS is a project dedicated to building a database of current and past documents in computing, librarianship, information science and technology, and related application activities. Retrieved: May 22, 2013. http://rclis.org/

SHERPA (2013). SHERPA is part of the JISC FAIR (Focus on Access to Institutional Resources) Retrieved: May 22, 2013. http:// www.sherpa.ac.uk/index.html

Suber, Peter (2012). Open Access News (Formerly FOS News). Retrieved: May 22, 2013. http://www.earlham.edu/ peters / fos/fosblog.html

Tajoli, Zeno (2005) METALIS, an OAI Service Provider., 2005 . 9th DELOS Network of Excellence Thematic Workshop, Heraklion (Crete), 11-13 May 2005. Retrieved: May 22, 2013. http://eprints.rclis.org/6209/ 